\title{
Apparition de symptômes de type "Green crinkle» après traitement mutagène appliqué à un clone de pommier contaminé par diverses maladies de dégénérescence
}

\author{
J. Lemoine et Y. Lespinasse \\ INRA, Station d'amélioration des espèces fruitières et ornementales, Beaucouzé, 49000 Angers, France
}

(reçu le 25 février 1988, accepté le 10 avril 1989)

\begin{abstract}
Résumé - Un traitement mutagène aux rayons gamma du cobalt 60 a été appliqué sur le clone de pommier "Golden Delicious" X551 T, déjà affecté par diverses maladies de dégénérescence, "Apple chlorotic leaf spot», "Apple stem grooving", "Apple spy epinasty and decline", "Apple stem pitting" et "Apple rubbery wood". Lors de l'étude des mutants obtenus, des symptômes de type "Green crinkle" ont été observés sur un arbre. Ces symptômes sont apparus sur les fruits d'une seule charpentière X551 MGC+; l'autre partie de l'arbre (X551 MGC-) ne présente pas de symptôme. La transmissibilité du symptôme et son rapprochement avec la maladie du "Green crinkle" sont mises en évidence. Une étude de l'état sanitaire des 2 parties de l'arbre révèle des différences qualitatives (apparition de "Green crinkle" chez X551 MGC+) et quantitatives (symptômes de «Rubbery wood» atténués chez X551 MGC+ et aggravés chez X551 MGC-). X551 MGC- inoculé par X551 MGC+ ne montre jamais de symptôme de "Green crinkle». Malgré les difficultés d'interprétation, une hypothèse explicative est proposée; des travaux sont en cours pour tester l'hypothèse avancée et tenter de répondre aux questions posées.
\end{abstract}

Malus domestica - maladies à virus - Green crinkle - mutagenèse - transmission virale

Summary - Appearance of "Green crinkle" type symptoms after mutagenic treatment of an apple tree clone affected by various degenerative diseases. A mutagenic treatment with Cobalt 60 gamma rays has been applied on a "Golden Delicious" apple clone X551 T, already infected by "Apple chlorotic leaf spot", "Apple stem grooving", "Apple spy epinasty and decline", "Apple stem pitting" and "Apple rubbery wood" diseases. During the study of the selected mutants, "Green crinkle" like symptoms were observed on a particular tree. These symptoms appeared on the fruits of a single scaffold X551 MGC+; the other part of the tree (X551 MGC-) had no symptom at all. The transmission of the symptom and its identification with the virus disease "Green crinkle" (GC) were established. A comparative study of the 2 parts of the tree showed qualitative (appearence of GC in X551 MGC+) and quantitative differences (symptoms of "Apple rubbery wood" decreased in X551 MGC+ and enhanced in X551 MGC-). X551 MGC- inoculated with X551 MGC+ did not show any symptoms of GC. Despite the difficulties in interpreting such results, a hypothesis has been put forward; studies are in progress to test this hypothesis and to try to answer the questions.

Malus domestica - virus diseases - Green crinkle - mutagenic treatment - virus disease transmission

\section{Introduction}

Les espèces à multiplication végétative, en particulier les arbres fruitiers, se prêtent bien à une amélioration par mutagenèse. II est en effet très facile, par greffage ou bouturage, de multiplier toute nouveauté. Chez le pommier, nous disposons d'une méthode d'irradiation de bourgeons à l'état dormant qui a donné de bons résultats (Decourtye et Lantin, 1971). L'intérêt de la muta- genèse est qu'un nombre limité de caractères peut être modifié sans trop altérer le reste du génotype; cette situation accroît donc les chances d'obtenir de nouveaux cultivars aux performances agronomiques et commerciales déjà confirmées. La principale difficulté est la formation de chimères, puisqu'une mutation affecte une cellule et que le matériel irradié est un apex pluricellulaire. La mise en évidence de ces chimères et leur stabilisation demandent du temps 
et de l'espace. Les mutations, spontanées ou provoquées, conduisent à des modifications qualitatives et quantitatives, tant au niveau de la structure de l'arbre que des qualités du fruit. Les mutations sans intérêt agronomique sont, en général, rapidement éliminées, soit en pépinière, soit lors des premières années de fructification.

Des travaux relativement anciens (Gilmer et Einset, 1959) décrivent des mutations chez le pommier, présentant un aspect proche de symptômes induits par des virus. Dans ce cas particulier, l'origine virale a pu être écartée; en effet, l'inoculation par lambeaux d'écorce n'a induit aucun symptôme chez les indicateurs. II s'agissait bien de mutations non associées à la présence de particules virales.

Dans le cadre des recherches en mutagenèse conduites à la Station d'amélioration des espèces fruitières et ornementales d'Angers, un clone de pommier, déjà affecté de diverses maladies d'origine virale, a été irradié. Sur l'un des mutants obtenus, des anomalies sur fruits sont apparues; elles sont proches des symptômes attribués à la maladie appelée «Green crinkle». Ce phénomène, limité à une seule charpentière du mutant, pourrait être le résultat du traitement.

Cet article rapporte les résultats de 3 essais destinés à étudier :

- la transmission du caractère observé après inoculation par greffage à du matériel sain;

- l'effet du traitement mutagène sur les organismes associés aux maladies de dégénérescence présentes au moment de l'irradiation;

- le comportement de chacune des 2 parties du mutant à l'égard du “Green crinkle».

\section{Matériel et Méthodes}

Des rameaux à bourgeons dormants de la variété de pommier "Golden Delicious" (clone X551 T) ont été soumis au rayonnement gamma du cobalt 60 (dose $40 \mathrm{~Gy}$ ) puis greffés sur des arbres indemnes de maladies de dégénérescence connues, selon la technique de multiplication des bourgeons irradiés (Decourtye et Lantin, 1971). Une seconde multiplication $V_{2}$ a été effectuée en pépinière sur porte-greffe clonal sain. Les arbres obtenus sont étudiés en verger et les mutants sélectionnés pour des caractères portant aussi bien sur le port de l'arbre que sur l'aspect des fruits. Les mutants ainsi isolés sont à nouveau multipliés $\left(V_{3}\right)$ pour être comparés en essai bloc (Fig. 1.1).

L'arbre étudié a été repéré dans l'un de ces essais comportant 8 mutants greffés sur porte-greffe MM106 indemne de virus, à raison d'un arbre par parcelle élémentaire et 10 répétitions.

Parmi les 10 arbres étudiés du mutant X551.1.34.4, l'un d'entre eux a révélé, sur les fruits de l'une des branches charpentières (X551 MGC+), des symptômes très proches sinon identiques à ceux provoqués par la maladie du “Green crinkle» (Kristensen, 1960); l'autre partie de l'arbre (X551 MGC-) ne présente aucun symptôme (Fig. 1.1). Les symptômes de «Green crinkle" sont visibles sur les jeunes fruits et sont caractérisés par des dépressions ou de petites crevasses; par la suite, la croissance des fruits étant irrégulière, ceuxci sont déformés et bosselés (Fig. 2). En fonction des conditions climatiques du printemps, le fond des dépressions est, soit lisse soit légèrement rugueux (symptôme de "Green crinkle»), soit craquelé avec une forte rugosité (symptôme de "Star crack"). L'observation de ces symptômes sur l'arbre initial a été confirmée au cours de 5 récoltes successives. Les symptômes sur fruits sont restés rigoureusement localisés à une seule charpentière de l'arbre; ce dernier n'est donc pas homogène pour le caractère étudié.

Le clone d'origine (X551 T) est contaminé par 5 maladies de dégénérescence («Apple chlorotic leaf spot virus" (CLSV), “Apple spy epinasty and decline" (SD), "Apple stem grooving" (SG), «Apple stem pitting" (SP) et «Apple rubbery wood" (RW), appelé par la suite "Bois caoutchouc»), mais ne présente pas de symptôme de "Green crinkle».

C'est à partir des 2 origines bien distinctes de l'arbre (X551 MGC+ et X551 MGC-) que nous avons prélevé le matériel pour l'étude. Le clone X551 T a été retenu comme témoin. Du fait que la maladie du "Green crinkle» ne s'exprime pas tous les ans avec la même fréquence et intensité (Lemoine, 1987), nous avons introduit en témoin de la maladie un clone de "Golden Delicious" contaminé par une souche de "Green crinkle" (témoin malade). A notre connaissan$c e$, il n'existe pas de souche "pure" de "Green crinkle" et ce témoin malade est aussi contaminé par du CLSV, SP, SD, RW.

\section{Essai 1 : Etude de la transmission du caractère observé après inoculation par greffage sur du matériel sain}

La variété "Lysgolden" (X2727), mutant de "Golden Delicious" indemne de rugosité naturelle et de maladies de dégénérescence connues, a été inoculée par X551 T, X551 MGC-, X551 MGC+ et le témoin malade. Ce mutant de "Golden Delicious" a été choisi pour mieux détecter la maladie du "Green crinkle" en années de faible expression des symptômes; en effet, certaines années les symptômes peuvent se limiter à une simple rugosité de l'épiderme, peu différente de la rugosité naturelle.

Le greffage des inoculums a été réalisé en mai 1978 , selon la technique de la "greffe en coulée", sur des arbres âgés de 4 ans greffés sur porte-greffe M26 (Fig. 1.2). Le choix de ces arbres âgés de 4 ans permet une observation des symptômes sur fruit 18 mois après inoculation.

Sur chaque arbre, 5 greffes ont été pratiquées et chaque traitement comportait 9 répétitions, soit 9 arbres. L'observation des arbres s'est poursuivie pendant 5 ans de 1979 à 1983.

Essai 2 : Etude de l'effet du traitement mutagène sur les organismes associés aux maladies de dégénérescence présents au moment de l'irradiation

Des tests biologiques ont été réalisés à partir de X551 T, X551 MGC- et X551 MGC+ sur la gamme d'indicateurs ligneux suivante : Malus platycarpa, Malus soulardii, “Spy 227", "Virginia Crab", “Lord Lambourne” 
1. 1. - Origine du materiel
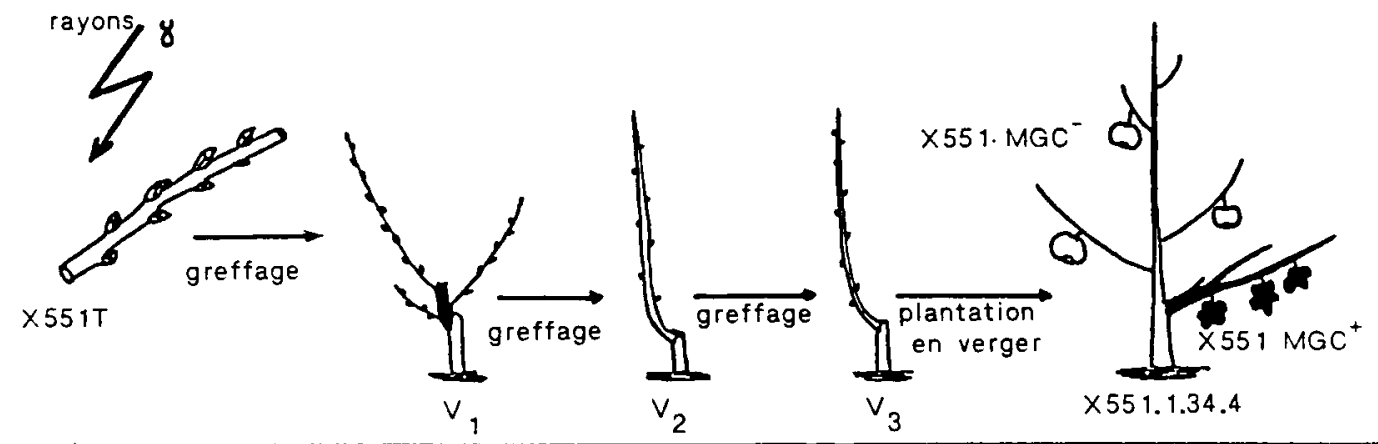

1. 2. - Essai 1 : Etude de la transmission du caractère observé

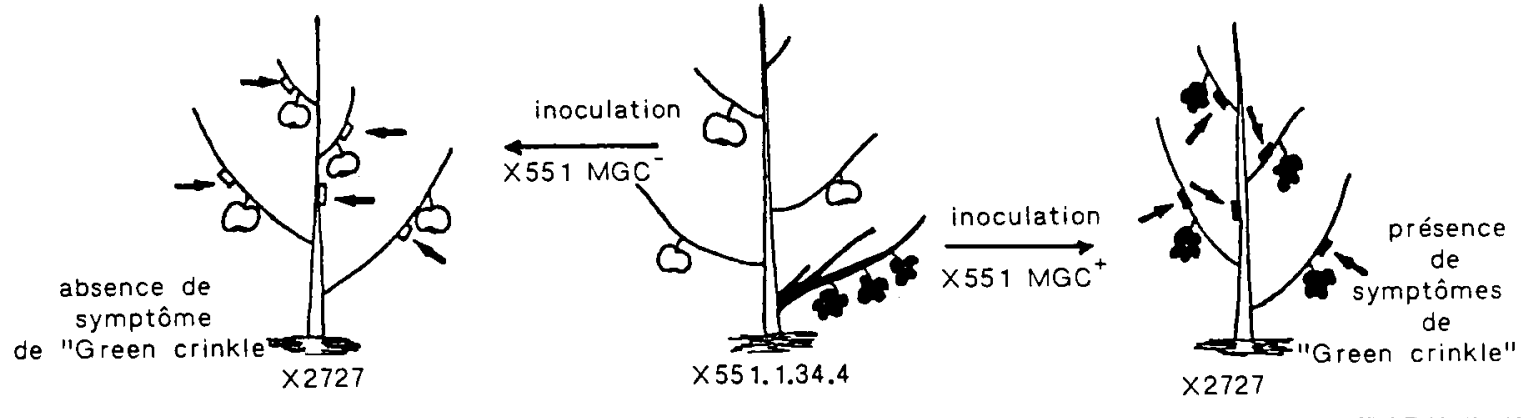

1.3. - Essai 3 : Réponses de chaque partie de l'arbre et du témoin à l'inoculation
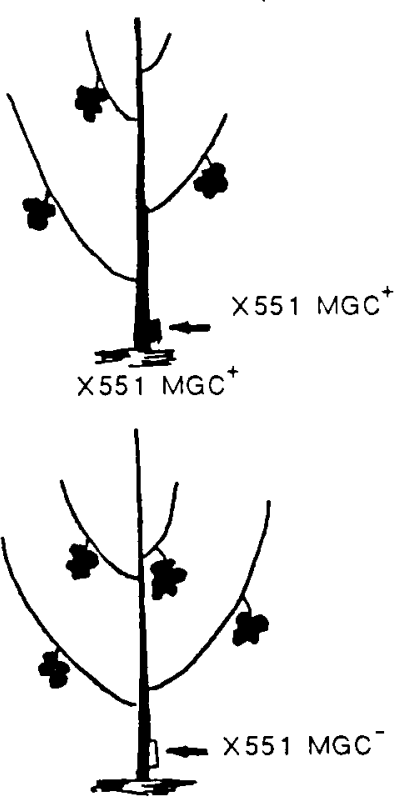

$\times 551 \mathrm{MGC}^{+}$

$\Phi=$ absence do symptôme

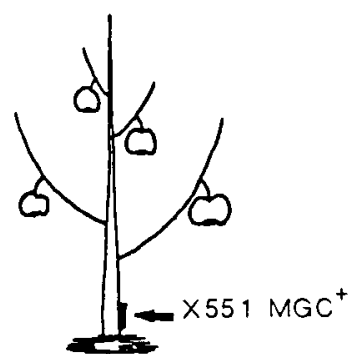

$\times 551 \mathrm{MGC}^{-}$

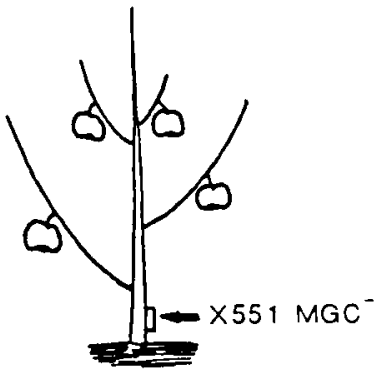

$\times 551 \mathrm{MGC}^{-}$

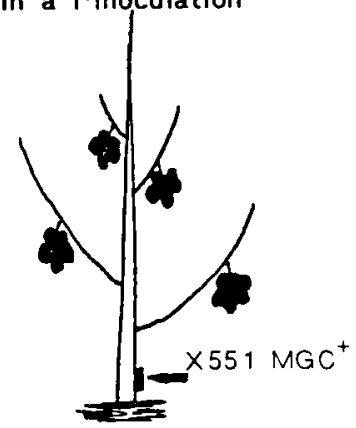

$\times 55, T, \times 972, \times 2727$

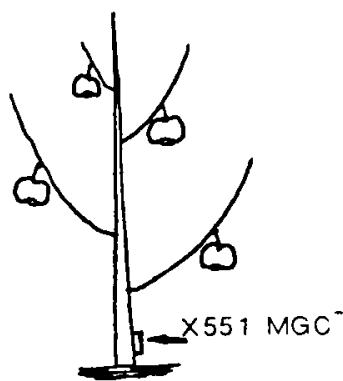

$\times 551 \mathrm{~T}, \times 972, \times 2727$

$=$ présence de symptômes

$\mathbf{D}-$ inoculation $\times 551 \mathrm{MGC}^{+}$

Fig. 1. Origine du matériel, protocole et résultats des essais 1 et 3.

et 2 clones de "Golden Delicious" : X551 T et le clone sain X972. La sensibilité de ces indicateurs aux maladies de dégénérescence est indiquée sur le Tableau I. L'inoculation a été pratiquée au mois d'août 1979 , selon la technique du udouble écussonnage». Les observations des symptômes ont été réalisées pendant 2 années. Une mensuration totale des pousses a été effectuée en fin de première année de croissance. A la suite des premiers résultats, les 3 clones étudiés ont à nouveau été indexés en 1985, uniquement sur l'indicateur "Lord Lambourne".

Essai 3 : Etude du comportement de chacune des deux parties du mutant à l'égard du «Green crinkle»

Cet essai avait pour but d'étudier et de comparer le comportement de X551 MGC-, X551 T, X972 et X2727 


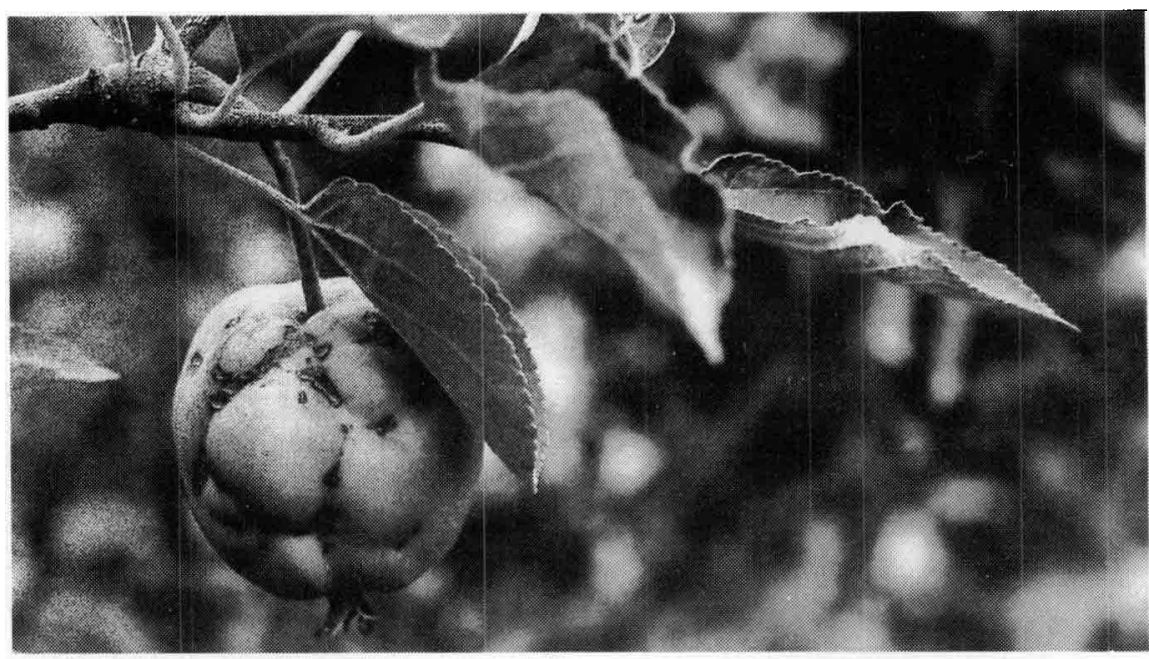

Fig. 2. Symptômes de type "Green crinkle" induit par X551 MGC+ à l'indicateur "Lysgolden".

après inoculation par X551 MGC+. Les clones X972 et X2727 ont été ajoutés pour disposer de témoins indemnes de virus. L'ensemble de ce matériel a été utilisé, soit pour produire la charpente de l'arbre, soit pour servir d'inoculum (Fig. 1.3), de manière à étudier toutes les interactions possibles (Tableau II).

Ces arbres ont été préparés en août 1979, plantés au cours de l'hiver 1980-1981 à raison de 4 arbres par traitement. L'observation des fruits a débuté en 1983.

\section{Résultats}

Sur l'ensemble des traitements de l'essai 1 , nous n'avons pas observé de symptôme sur fruits, l'année même de l'inoculation. Au cours des 5 récoltes suivantes, les inoculums X551 T et X551 MGC- n'ont pas induit de symptôme de "Green crinkle» (Tableau III et Fig. 1.2). Pendant cette

Tableau I. Comportement des indicateurs à l'égard des maladies de dégénérescence du pommier.

\begin{tabular}{|c|c|c|c|c|c|c|c|c|c|c|}
\hline \multirow[t]{2}{*}{ Indicateurs } & \multirow[b]{2}{*}{$C L S V$} & \multirow[b]{2}{*}{$\begin{array}{c}\text { Spy } \\
\text { décline }\end{array}$} & \multirow[b]{2}{*}{$\begin{array}{l}\text { Stem } \\
\text { pitting }\end{array}$} & \multirow[b]{2}{*}{$\begin{array}{c}\text { Stem } \\
\text { grooving }\end{array}$} & \multirow[b]{2}{*}{$\begin{array}{l}\text { Rubbery } \\
\text { wood }\end{array}$} & \multirow[b]{2}{*}{ Mosaic } & \multirow[b]{2}{*}{ Prolifération } & \multicolumn{3}{|c|}{ Maladies sur fruits } \\
\hline & & & & & & & & $\begin{array}{l}\text { Green } \\
\text { crinkle }\end{array}$ & $\begin{array}{c}\text { Russet } \\
\text { ring }\end{array}$ & $\begin{array}{c}\text { Russet } \\
\text { wart }\end{array}$ \\
\hline Malus platycarpa & + & - & - & - & - & - & - & - & + & - \\
\hline Malus soulardii & + & - & - & - & - & - & - & + & - & - \\
\hline Spy 227 & + & + & + & - & - & - & - & - & - & - \\
\hline Virginia crab & - & + & + & + & - & - & - & - & - & - \\
\hline $\begin{array}{l}\text { Lord lambourne } \\
\text { Golden } \\
\quad \text { X551 T }\end{array}$ & - & - & - & - & + & + & - & - & - & - \\
\hline $\begin{array}{l}\times 972 \\
\times 2727\end{array}$ & - & - & - & - & - & + & + & + & + & + \\
\hline
\end{tabular}

+ = réaction positive : expression des symptômes de la maladie; $-=$ réaction négative.

Tableau II. Essai 3 : les diverses associations réalisées.

Charpente de l'arbre (19r greffage) Inoculum (2e greffage)

X551 $\quad \times 551 \mathrm{MGC}^{+} \quad \times 551 \mathrm{MGC}^{-} \quad \times 2727 \quad \times 972$

$\times 551 \mathrm{~T}$

$\times 551 \mathrm{MGC}+$

X 551 MGC-

$\times 2727$

$\times 972$

+
+
+
+
+

+
+
+
+
+


Tableau III. Essai 1 : production moyenne de la variété “Lysgolden” après inoculation par les clones étudiés et pourcentage de fruits présentant des symptômes de "Green crinkle".

Traitements

Production moyenne en $\mathrm{kg} / \mathrm{arbre}$

$\begin{array}{lllll}1979 & 1980 & 1981 & 1982 & 1983\end{array}$

Pourcentage de fruits avec symptômes

Cumul

$\begin{array}{lllll}1979 & 1980 & 1981 & 1982 \quad 1983\end{array}$

$\begin{array}{lccccccccccc}\text { Témoin «malade» } & 2,7 & 3,4 & 4,6 & 15,4 & 19,2 & 45,3 \mathrm{~b} * & 69,2 & 98,1 & 98,3 & 4,7 & 48,1 \\ \text { X 551 MGC+ } & 2,1 & 1,3 & 2,5 & 12,9 & 17,3 & 36,1 \mathrm{c} & 67,4 & 92,5 & 87,2 & 4,6 & 50,3 \\ \text { X 551 MGC- } & 2,5 & 3,2 & 3,4 & 16,1 & 20,3 & 45,5 \mathrm{~b} & 0 & 0 & 0 & 0 & 0 \\ \text { X 551 T } & 3,4 & 3,8 & 5,3 & 17,5 & 22,2 & 52,2 \mathrm{a} & 0 & 0 & 0 & 0 & 0\end{array}$

* Les chiffres suivis de la même lettre ne sont pas significativement differents au seuil $5 \%$ (test de Duncan).

même période, l'inoculum $\times 551 \mathrm{MGC}+$ et le «témoin malade» ont induit des symptômes de "Green crinkle". En fonction des conditions climatiques du printemps, les symptômes s'expriment avec plus ou moins d'intensité (Lemoine, 1987); en 1982, les températures élevées du printemps ont été peu favorables à l'expression de la maladie. L'aspect des symptômes induits par X551 MGC+ et par le «témoin malade», est identique; le pourcentage de fruits atteints est très voisin (Tableau III). Cela met bien en évidence que le symptôme exprimé par X551 MGC+ est transmissible et qu'il correspond aux symptômes de la maladie appelée "Green crinkle». Les arbres inoculés par $\times 551 \mathrm{MGC}+$ ont une produc- duction cumulée faible sur les 5 années. Ces différences sont significativement différentes (test de Duncan) de celles des arbres inoculés par X551 T et X551 MGC- (Tableau III).

L'indexage réalisé en pépinière (essai 2) sur divers indicateurs, montre que l'ensemble du matériel testé (X551 T, X551 MGC+, X551 MGC-) est contaminé par les mêmes maladies de dégénérescence (CLSV, SP, SD, SG, RW) (Tableau IV). A la fin de la première année de croissance (Tableau V), nous observons que :

- l'inoculum X551 MGC- induit une réduction de la vigueur des indicateurs par rapport à l'inoculum X551 T et X551 MGC+, à l'exception de l'indicateur X551 T, déjà contaminé;

Tableau IV. Essai 2 : réponse des indicateurs après inoculation par les clones étudiés.

\begin{tabular}{|c|c|c|c|c|c|c|c|c|}
\hline & $\begin{array}{l}\text { Lord } \\
\text { Lambourne }\end{array}$ & Spy 227 & & $\begin{array}{c}\text { Malus } \\
\text { platycarpa }\end{array}$ & $\begin{array}{l}\text { Malus } \\
\text { soulardii }\end{array}$ & $\begin{array}{c}\text { Golden } \\
\times 551 \text { et } \times 972\end{array}$ & $\begin{array}{l}\text { Virginia } \\
\text { crab }\end{array}$ & \\
\hline$\times 551 \mathrm{~T}$ & Rubbery wood ++ & $\begin{array}{l}\text { CLSV } \\
\text { Spy decline }\end{array}$ & $\begin{array}{l}-1 \\
++\end{array}$ & CLSV ++ & CLSV +++ & $-{ }^{*}$ & $\begin{array}{l}\text { Stem pitting } \\
\text { Stem grooving }\end{array}$ & $\begin{array}{l}+++ \\
+\end{array}$ \\
\hline X $551 \mathrm{MGC}^{-}$ & Rubbery wood +++ & $\begin{array}{l}\text { CLSV } \\
\text { Spy decline }\end{array}$ & $\begin{array}{l}\text { t++ } \\
+++\end{array}$ & CLSV +++ & CLSV +++ & - & $\begin{array}{l}\text { Stem pitting } \\
\text { Stem grooving }\end{array}$ & $\begin{array}{l}+++ \\
+\end{array}$ \\
\hline X $551 \mathrm{MGC}^{+}$ & Rubbery wood + & $\begin{array}{l}\text { CLSV } \\
\text { Spy decline }\end{array}$ & $\begin{array}{l}++ \\
++\end{array}$ & CLSV ++ & CLSV ++ & - & $\begin{array}{l}\text { Stem pitting } \\
\text { Stem grooving }\end{array}$ & $\begin{array}{l}+++ \\
+++\end{array}$ \\
\hline
\end{tabular}

CLSV = Chlorotic Leaf Spot Virus; + = le nombre de + indique l'importance du symptôme; * = les symptômes sur fruit n'ont pas été observés car les plants ont été arrachés avant la $1^{r e}$ fructufication.

Tableau V. Essai 2 : longueur totale en $\mathrm{cm}$ des pousses des indicateurs un an après inoculation (moyenne de 10 répétitions par traitement) et longueur exprimée en \% de $\times 551 \mathrm{~T}$.

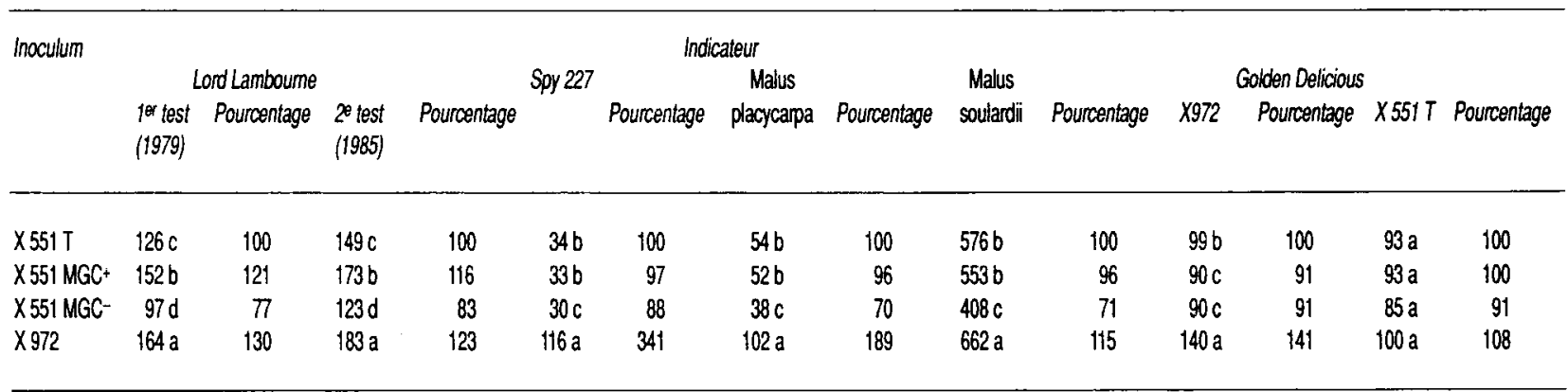


- dans le cas de l'indicateur "Lord Lambourne", les 2 parties de l'arbre (X551 MGC+ et X551 MGC-) ont, par rapport à X551 T, un comportement significativement différent. Ainsi, avec l'inoculum X551 $\mathrm{MGC}^{+}$, la vigueur de l'indicateur "Lord Lambourne" est forte; elle est nettement réduite après inoculation avec X551 MGC-. Ce phénomène a été confirmé au cours du second test en 1985;

- de même, l'intensité de l'expression du «Stem grooving" est plus élevée chez X551 MGC+ (Tableau IV).

Les observations enregistrées en pépinière et au verger montrent que le clone $\times 551 \mathrm{MGC}^{+}$a une vigueur supérieure aux clones $\times 551 \mathrm{~T}$ et X551 MGC- (Tableau VI). Ces résultats sont le reflet de la différence d'état sanitaire de ces clones vis-à-vis du "Bois caoutchouc", dont la présence altère nettement la vigueur de l'arbre.

En verger (essai 3) au cours des 4 années de fructification, nous avons observé un comportement différent des clones étudiés (Tableau VII et Fig. 1.3). Lorsque la charpente de l'arbre est constituée par X551 T, X2727 ou X972, seul l'inoculum X551 MGC+ induit des symptômes, ce qui confirme la transmissibilité du caractère. Les premières années, le faible taux de fruits présentant des symptômes est le reflet de l'évolution lente de la maladie dans l'arbre.

Conformément à ce qui avait été observé sur l'arbre initial, les fruits de la partie X551 MGCn'expriment pas de symptôme en présence de l'inoculum X551 MGC+.

En 1985, le réindexage des arbres issus du clone X551 MGC- a montré que les 5 maladies sont toujours présentes mais que la maladie du «Green crinkle» n'est toujours pas détectée.

\section{Discussion et Conclusion}

Les symptômes de type "Green crinkle" observés sur les fruits d'une des branches charpentières du mutant X551.1.34.4 sont transmissibles par greffage aux clones X551 T, X972 et X2727, confirmant ainsi la nature infectieuse du phénomène. II faut préciser que le mutant X551.1.34.4 provient du clone X551 T déjà contaminé par 5 maladies de dégénérescence mais n'exprimant jamais de symptômes de "Green crinkle».

L'analyse sanitaire, effectuée par des tests biologiques sur hôtes ligneux, montre que le clone X551 MGC+ porte les mêmes maladies de dégénérescence que X551 MGC-, mais que la souche de "Bois caoutchouc" est atténuée. Cela se traduit par une croissance forte du clone X551 $\mathrm{MGC}^{+}$en pépinière et une bonne vigueur de l'indicateur "Lord Lambourne" après inoculation.

Le clone X551 MGC+ manifeste des symptômes identiques à ceux de la maladie du "Green crinkle" et provoque, après inoculation de X551 T, les mêmes symptômes; ceux-ci n'ont jamais été observés chez X551 MGC-.

Ces observations laissent supposer que le traitement mutagène appliqué sur X551 T a provoqué 2 modifications distinctes:

- l'une, au niveau des maladies de dégénérescence : apparition des symptômes de «Green crinkle", diminution ou augmentation de l'expression de la maladie du "Bois caoutchouc»;

- l'autre, au niveau de l'hôte par la révélation d'un clone, X551 MGC-, ne manifestant aucun symptôme après inoculation par le «Green crinkle".

Ces résultats sont difficiles à interpréter et nécessitent l'examen de la situation, d'une part, chez l'agent infectieux et d'autre part, chez l'hôte.

\section{L'agent infectieux}

On ne connaît pas l'identité de l'agent responsable du "Green crinkle». Est-ce un virus, un mycoplasme, un complexe de particules infectieuses ? Quelles relations peut avoir cet agent avec ceux des maladies de dégénérescence décelées et conduisant aux symptômes du «Bois caoutchouc", du CLSV, du "Spy epinasty and

Tableau VI. Vigueur en pépinière et en verger des clones étudiés, exprimée par la circonférence des troncs en mm (10 arbres par traitement).

1980

(pépinière)
1981

1982

141,5

137,5 a

$129,2 \mathrm{~b}$

$116,4 \mathrm{c}$

\section{7,5}

$80,0 \mathrm{~b}$

$77,7 \mathrm{~b}$
1983

\section{$X 972$ (témoin sain) \\ $\times 551$ MGC+ \\ $\times 551 \mathrm{~T}$ \\ X 551 MGC-}

$$
\begin{aligned}
& 58,9 \\
& 55,4 \text { a } \\
& 51,2 \text { b } \\
& 47,2 \text { c }
\end{aligned}
$$

214,1

199,6 a

$180,6 \mathrm{~b}$

$169,5 \mathrm{~b}$

\footnotetext{
* les chiffres suivis de la même lettre ne sont pas significativement différents au seuil $5 \%$ (test de Duncan).
} 
Tableau VII. Essai 3 : pourcentage de fruits manifestant des symptômes de “Green crinkle».

\begin{tabular}{|c|c|c|c|c|c|c|}
\hline \multirow{2}{*}{$\begin{array}{l}\text { Charpente } \\
\text { de l'arbre }\end{array}$} & & \multicolumn{3}{|c|}{ Inoculum } & \multirow[b]{2}{*}{$\times 2727$} & \multirow[b]{2}{*}{ X972 } \\
\hline & & $X 551 M G C^{+}$ & X551 MGC- & $\times 551 T$ & & \\
\hline \multirow{5}{*}{ X551 MGC+ } & 1983 & 21 & 17 & 17 & 19 & 19 \\
\hline & 1984 & 23 & 18 & 19 & 21 & 23 \\
\hline & 1985 & 82 & 86 & 84 & 81 & 86 \\
\hline & 1986 & 80 & 89 & 81 & 89 & 85 \\
\hline & 1987 & 85 & 90 & 86 & 90 & 86 \\
\hline \multirow{5}{*}{ X551 MGC- } & 1983 & 0 & 0 & 0 & 0 & 0 \\
\hline & 1984 & 0 & 0 & 0 & 0 & 0 \\
\hline & 1985 & 0 & 0 & 0 & 0 & 0 \\
\hline & 1986 & 0 & 0 & 0 & 0 & 0 \\
\hline & 1987 & 0 & 0 & 0 & 0 & 0 \\
\hline \multirow{5}{*}{ X551 T } & 1983 & 0 & 0 & 0 & 0 & 0 \\
\hline & 1984 & 15 & 0 & 0 & 0 & 0 \\
\hline & 1985 & 25 & 0 & 0 & 0 & 0 \\
\hline & 1986 & 22 & 0 & 0 & 0 & 0 \\
\hline & 1987 & 61 & 0 & 0 & 0 & 0 \\
\hline \multirow{5}{*}{ X2727 } & 1983 & 0 & 0 & 0 & 0 & 0 \\
\hline & 1984 & 23 & 0 & 0 & 0 & 0 \\
\hline & 1985 & 56 & 0 & 0 & 0 & 0 \\
\hline & 1986 & 24 & 0 & 0 & 0 & 0 \\
\hline & 1987 & 63 & 0 & 0 & 0 & 0 \\
\hline \multirow{5}{*}{ X972 } & 1983 & 0 & 0 & 0 & $-{ }^{\star}$ & 0 \\
\hline & 1984 & 21 & 0 & 0 & - & 0 \\
\hline & 1985 & 19 & 0 & 0 & - & 0 \\
\hline & 1986 & 20 & 0 & 0 & - & 0 \\
\hline & 1987 & 72 & 0 & 0 & - & 0 \\
\hline
\end{tabular}

- combinaison non réalisée.

decline", de l'“Apple stem pitting", de l'«Apple stem grooving" ? Peut-on imaginer qu'il y a entre eux compétition, synergie pour leur multiplication, leur diffusion, leur pouvoir pathogène?

Quel effet a pu avoir le traitement mutagène sur l'ensemble viral contenu dans X551 T ? Peuton imaginer une (ou des) mutation(s) exacerbant, d'une part, l'agent du «Bois caoutchouc» chez X551 MGC- et favorisant, d'autre part, l'expression de la maladie du "Green crinkle» chez X551 $\mathrm{MGC}+$ ?

Existe-t-il un lien chez X551 MGC+ entre la diminution significative de l'agressivité du «Bois caoutchouc» et l'apparition des symptômes de "Green crinkle"? Pour tester cette dernière hypothèse, un essai a été mis en place en 1988: X972 a été inoculé par un complexe CLSV, SP, $\mathrm{SD}, \mathrm{SG}$ associé à des souches de "Bois caoutchouc» d'agressivité différente.

Enfin, peut-on imaginer qu'une certaine entité virale puisse évoluer par mutation et produire des symptômes différents?
II est difficile de répondre à ces questions. On peut toutefois formuler l'hypothèse suivante : le clone X551 T possède une souche de "Green crinkle» $(\mathrm{gc})$ très faible; elle ne s'exprime jamais. Le traitement mutagène aurait modifié la souche gc en souche GC, qui se multiplie, s'exprime naturellement chez X551 MGC+ et provoque après inoculation, des symptômes sur X551 T mais pas sur X551 MGC-. Cette différence entre X551 MGC+ et X551 MGC- pourrait être due à des modifications inhérentes à l'effet du traitement mutagène sur l'hôte.

\section{L'hôte}

L'hôte est bien muté puisque distinct de X551 T. II présente des fruits de maturité plus précoce et une production plus élevée. Peut-on imaginer que l'arbre étudié est en chimère ? On admet depuis Dermen (1951) que le méristème de pommier est constitué de 3 couches histogénétiques. Les couches $\mathrm{LI}$ et LII constituent préférentiellement les tissus externes jusqu'à l'assise cam- 
biale, alors que la couche LIII constitue les tissus internes (xylème et moelle). Le génotype X551 MGC- pourrait être en chimère avec en couche LIII, non apparente, le génotype X551 MGC+.

La branche charpentière X551 MGC+ serait issue d'un bourgeon différencié à partir de l'assise cellulaire profonde LIII. II est possible de formuler l'hypothèse génétique suivante : le clone de départ X551 T a les 3 assises génératrices LI, LII, LIII de génotype a/a; après traitement mutagène, on obtient le mutant X551.1.34.4 dont le génotype est modifié en $\mathrm{A} / \mathrm{a}$ pour les couches $\mathrm{LI}$ et LII, la couche LIII (X551 MGC+) étant identique au témoin (X551 T) de génotype a/a. Ainsi, le clone X551 MGC+ issu de la couche LIII a un génotype homogène $a / a$ dans toutes ses couches (Fig. 2).

Nous nous trouvons donc en présence des clones suivants :

- le clone de départ X551 T de génotype a/a; il possède une souche de "Green crinckle" (gc) faible qui ne s'exprime jamais. Inoculé avec le "témoin malade», il manifeste clairement des symptômes de "Green crinkle";

- le clone muté X551 MGC- en chimère et de génotype $\mathrm{A} / \mathrm{a}$; cette constitution génotypique en LI et LII empêche la manifestation des symptômes de "Green crinkle»; il porte en LIII le génotype $a / a$;

- le clone X551 MGC+, issu de la couche LIII de X551 MGC-, de génotype a/a homogène; il manifeste des symptômes de "Green crinkle» du fait de la présence de la souche GC issue par mutation de la souche non agressive gc.

II est possible de révéler artificiellement la couche profonde LIII par la néoformation de bourgeons à partir de racines (Decourtye, 1987). En 1988, le génotype X551 MGC- a été bouturé; les racines obtenues seront isolées pour provoquer l'apparition des drageons de constitution homogène et de type LIII. La présence ou l'absence de symptômes sur fruits permettra de tester l'hypothèse avancée d'une constitution en chimère de l'arbre étudié X551.1.34.4.

On peut avancer d'autres hypothèses pour expliquer le phénomène observé sur X551.1.34.4.

La répartition des agents infectieux au sein d'une même plante est souvent inégale. Cela a été démontré chez le pommier à propos du «Tomato ring spot virus» (Bitterlin et al., 1984) ou du "Chlorotic leaf spot virus" (Frindlund, 1983). $\mathrm{Ce}$ fait fut aussi rapporté chez les plantes annuelles. Ainsi, Lecoq et al. (1982) observent simultanément sur certains plants de piment des secteurs malades et des secteurs sains pendant une longue période.

Par ailleurs, il est possible d'envisager un phénomène de prémunition entre "souches" localisées dans différentes parties de la plante (Marchoux, 1987). Cette hypothèse expliquerait la protection de X551 MGC- vis-à-vis de X551 $\mathrm{MGC}^{+}$. Cela suppose que la souche gc contenue dans X551 T ait muté vers une souche gc $^{*}$ prémunisante, portée par X551 MGC-, mais non décelable par indexage, et une souche forte GC qui induit des symptômes chez X551 MGC+.

Un essai a été mis en place pour tester l'effet "prémunisant" du clone X551 MGC- vis-à-vis des souches isolées des variétés "Granny Smith", "Idared", "Golden Delicious".

II est aussi possible que le clone X551 MGCpuisse véhiculer les particules infectieuses, sans permettre l'expression du "Green crinkle", et en empêcher la multiplication. Ce phénomène a été mis en évidence par Cousin (1977) dans le cas de la résistance des pois au virus de la «Mosaïque commune» du pois. L'auteur démontre que cette résistance est une immunité empêchant toute multiplication de virus, mais

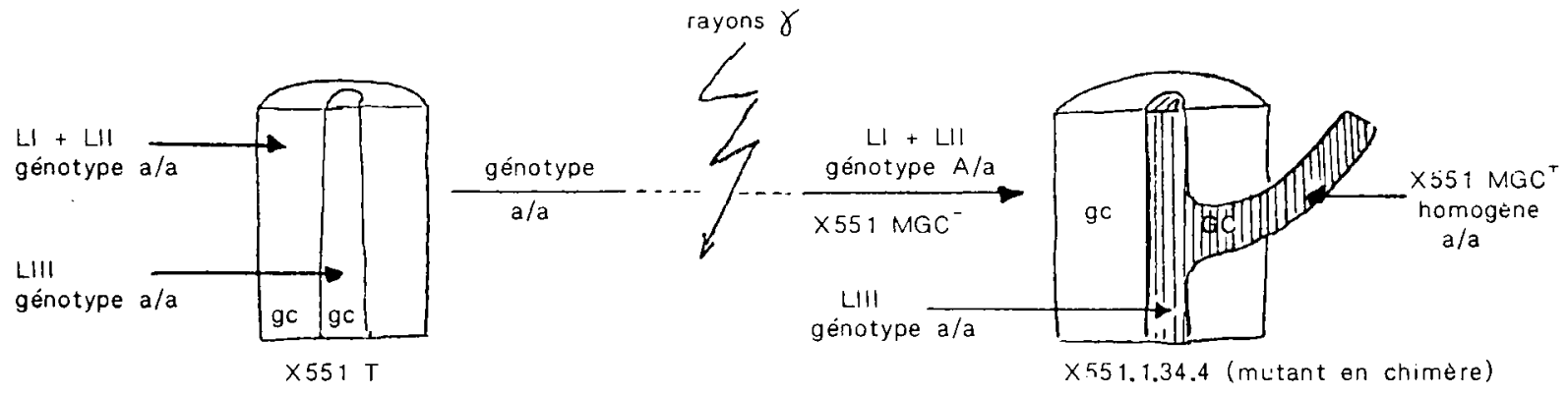

$g c=$ souche non agressive de "Green crinkle"
$G C=$ souche agressive de "Green crinkie"

Fig. 3. Hypothèse de la modification de l'hôte et de l'agent pathogène après traitement mutagène. 
permettant néanmoins la transmission de l'information génétique virale. Pour tester cette hypothèse, nous avons créé des arbres à 3 composantes en greffant successivement X551 $\mathrm{MGC}^{+}$ puis X551 MGC- et X972. Ce matériel est en cours d'observation.

A notre connaissance, c'est la première fois que l'on envisage d'attribuer une différence qualitative (absence ou présence de symptômes) à une constitution tissulaire en chimère. Cette hypothèse est aussi avancée pour expliquer le phénomène "plage verte-plage jaune» constituant certaines Mosaïques d'origine virale (Matthews, 1981; Marchoux, 1987).

Il est à noter que nous avons observé un autre cas de modification dans l'expression des symptômes de "Flat limb" (induit par du "Bois caoutchouc») après traitement mutagène d'un clone de la variété "Melrose" contaminée par diverses maladies de dégénérescence. Les résultats obtenus seront à comparer avec ceux que nous venons de décrire.

\section{Remerciements}

Nous remercions les lecteurs, F. Dosba et G. Marchoux, pour les corrections apportées et les suggestions émises pour améliorer le manuscrit.

\section{Références}

Bitterlin M.W., Gonsalves D. \& Cummins J.N. (1984) Irregular distribution of Tomato Ringspot virus in apple trees. Plant Dis. 68, 567-571
Cousin R. (1977) Origine de la résistance du pois aux virus de la Mosaïque commune du pois. Le Tocsin du Radiateur 2, 85-86

Decourtye L. (1987) L'origine ontogénique des racines, puis des bourgeons néoformés sur des chimères de poirier (Pyrus communis L.) et de pommier (Malus pumila Mill.). Agronomie 7, 27-32

Decourtye L. \& Lantin B. (1971) Considérations méthodologiques sur l'isolement de mutants provoqués chez le pommier et le poirier. Ann. Amelior. Plant. (Paris) 21, 29-44

Dermen H. (1951) Ontogeny of tissues in stem and leaf of cytochimeral apples. Am. J. Bot. 38, 753-760

Fridlund P.R. (1983) Distribution of Chlorotic leaf spot virus infected buds on various lengths of apple budsticks in successive years. Acta Hortic. 130, 85-87

Gilmer R.M. \& Einset J. (1959) Some bud mutations of apple with virus like symptoms. Plant Dis. Rep. 43, 264-269

Kristensen R.H. (1960) Apple green crinkle. Virus disease of apples and pears. Technical communication 30, 33-34

Lecoq H., Pochard E., Pitrat M., Laterrot H. \& Marchoux G. (1982) Identification et exploitation de résistances aux virus chez les plantes maraîchères. Cryptogam. Mycol. 3, 333-345

Lemoine J. (1987) Incidence de l'action chaleur sur les symptômes induits par la maladie du "Green crinkle" sur le cultivar de pommier "Golden Delicious" (M. $x$ domestica Borkh.). Agronomie 7, 431-435

Marchoux G. (1987) Interactions entre virus chez un hôte commun. I. Interférences négatives, antagonismes, prémunition, résistance induite. Agronomie 7, 149-162

Matthews R.E.F. (1981) V. Leaf ontogeny and mosaic disease. In: Plant Virology (2nd ed.). Academic Press, New York, pp. 417-426 